\title{
Averaged control and observation of parameter-depending wave equations
}

\author{
Martin Lazar a , Enrique Zuazua ${ }^{\mathrm{b}, \mathrm{c}}$ \\ ${ }^{a}$ University of Dubrovnik, Department of Electrical Engineering and Computing, Ćira Carića 4, 20 000 Dubrovnik, \\ Croatia \\ ${ }^{\mathrm{b}}$ BCAM - Basque Center for Applied Mathematics, Alameda Mazarredo 14, E-48009 Bilbao, Basque Country, Spain \\ ${ }^{\mathrm{c}}$ Ikerbasque - Basque Foundation for Science, Alameda Urquijo 36-5, Plaza Bizkaia, 48011, Bilbao, Basque Country, \\ Spain \\ Received *****; accepted after revision +++++ \\ Presented by
}

\begin{abstract}
We analyze the problem of averaged observability and control of wave equations.

This topic is motivated by the control of parameter-dependent systems of wave equations. We look for controls ensuring the controllability of the averages of the states with respect to the parameter. This turns out to be equivalent to the problem of averaged observation in which one aims at recovering the energy of the initial data of the adjoint system by measurements done on its averages, under the assumption that the initial data of all the components of the adjoint system coincide.

The problem under consideration is weaker than the classical notion of simultaneous observation and control.

The method of proof uses propagation arguments based on H-measures or microlocal defect measures that reduce the problem to non-standard unique-continuation issues.

Using transmutation techniques we also derive some results on the averaged observation and control of parameter-dependent heat equations.

To cite this article: M. Lazar, E. Zuazua, C. R. Acad. Sci. Paris, Ser.
\end{abstract}

\section{Résumé}

Contrôle et observation en moyenne d'équations des ondes dépendant de paramètres.

On étudie le problème de l'observation et contrôle en moyenne d'équations des ondes.

Le sujet est motivé par le contrôle d'équations des ondes dépendant de paramètres. On s'intéresse à la contrôlabilité des moyennes des états par rapport aux paramètres. Ceci équivaut au problème de l'observation des états adjoints dépendant des paramètres mais tous avec les mêmes données initiales et ceci à partir de l'observation des moyennes.

Le problème en considération est plus faible que celui de la contrôlabilité ou observabilité simultanée étudié auparavant.

La méthode de preuves utilise des arguments de propagation qui emploient les H-mesures ou mesures de défaut microlocales, et qui réduisent le problème à des questions nouvelles de continuation unique. 
En utilisant des arguments de transmutation nous obtenons aussi quelques résultats pour le contrôle et l'observation en moyenne des équations paraboliques dépendant de paramètres.

Pour citer cet article: M. Lazar, E. Zuazua, C. R. Acad. Sci. Paris, Ser.

\section{Introduction}

We analyze the problem of controlling the averaged value of a system of parameter-depending wave equations by a single control. The problem is relevant in applications in which the control has to be chosen independently of the parameter value, in a robust manner.

This notion was previously introduced in [12] both in the context of finite-dimensional linear and PDE systems.

The problem is equivalent to that of averaged observability in which we try to determine the energy of an initial datum for the parameter-dependent wave equations, by means of simply observing their averages with respect to the parameter.

These notions are weaker than those of simultaneous control and observation ([6] and [1]). In simultaneous control all the wave equations, regardless of the value of the parameter, need to be controlled by the same control. In simultaneous observation the initial data of the solutions whose average is observed are supposed to depend arbitrarily on the parameter and not all to be the same as when dealing with averaged observation.

Our main results of averaged observation and, by duality, of averaged control, employ tools of microlocal analysis, and, more precisely, propagation arguments based on the use of microlocal defect measures or H-measures introduced independently by P. Gérard [9] and L. Tartar [11]. ${ }^{1}$ We refer the reader to the mentioned articles for the properties of these measures (localization, propagation, etc.) used in this Note. Our methods are strongly inspired in those developed in earlier works for the observation and control of hyperbolic equations $[3,4]$ and [6], among others.

In the next section we discuss the simplest case of a system of two distinct wave equations. Results on simultaneous observability, together with the appropriate assumptions and relations to the existing results are given in Section 3. Using transmutation techniques the results obtained for wave equations are then transferred to systems of heat equations (Section 4). We close the paper by pointing towards some open problems and future directions of research.

\section{Averaged observability}

As mentioned above, we consider the case where the system under consideration only involves two modes, depending on the velocity of propagation of solutions, denoted respectively by $c_{1}, c_{2}$ and $u_{1}, u_{2}$ :

$$
\begin{aligned}
\partial_{t t} u_{i}-\operatorname{div}\left(c_{i}(\mathbf{x}) \nabla u_{i}\right) & =0, \quad(t, \mathbf{x}) \in \mathbf{R}^{+} \times \Omega \\
u_{i}(0, \cdot) & =u^{0} \in \mathrm{L}^{2}(\Omega) \\
\partial_{t} u_{i}(0, \cdot) & =\tilde{u}^{0} \in \mathrm{H}^{-1}(\Omega), \quad i=1,2,
\end{aligned}
$$

Email addresses: martin.lazar@unidu.hr (Martin Lazar), zuazua@bcamath.org (Enrique Zuazua).

1. In the sequel, for simplicity, we shall use the terminology of H-measures. 
where the space domain $\Omega$ is assumed to be a compact manifold without boundary. As for the coefficients entering the system, we assume they are bounded from below by a positive constant. Furthermore, if not stated otherwise, it is assumed that $c_{1}$ is of class $\mathrm{C}^{1,1}$, thus ensuring well posedness of the bicharacteristic flow of the corresponding wave operator, while $c_{2}$ is merely continuous.

We investigate the conditions under which one can recover the energy of the initial data, which are the same for both components, by observing the average of solutions, $\theta u_{1}+(1-\theta) u_{2}$, with a parameter $\theta \in\langle 0,1]$.

The main result of this Note is as follows:

Theorem 2.1 Suppose the equations' coefficients satisfy

$$
c_{1}(\mathbf{x})-c_{2}(\mathbf{x}) \neq 0, \quad \mathbf{x} \in \omega
$$

where $\omega$ is an open subset of $\Omega$ and $T$ is a time such that $\langle 0, T\rangle \times \omega$ satisfies the Geometric Control Condition (GCC, [5]) for the first equation.

Then, for any $\theta \in\langle 0,1]$ there exists a constant $C_{\theta}$ such that the following estimate holds

$$
E(0):=\left\|u^{0}\right\|_{\mathrm{L}^{2}}^{2}+\left\|\tilde{u}^{0}\right\|_{\mathrm{H}^{-1}}^{2} \leq C_{\theta} \int_{0}^{T} \int_{\omega}\left|\theta u_{1}+(1-\theta) u_{2}\right|^{2} d \mathbf{x} d t .
$$

Remark 1 Several remarks are in order.

- The case $\theta=1$ holds trivially, as it coincides with the observability problem for a single equation, and corresponds to the well known result of [5].

- The theorem also holds, as well as its proof, if the system (1) is considered on a bounded, smooth domain $\Omega$ with Dirichlet boundary conditions. Indeed, the localization property for H-measures used in the proof holds within any open subdomain and is not affected by boundaries. Moreover, the propagation property of these measures also holds true in this case thanks to the results of [3]. The reflection law of measures is simpler to achieve and is more intuitive when the reflection is hyperbolic. In other words, a measure that vanishes along a bicharacteristic ray when getting to the boundary transversally is reflected as being null along the reflected broken bicharacteristic. This allows fully extending our results to the case of convex domains. For general domains one needs to consider glancing and diffractive situations as well.

Of course, all these technical difficulties disappear if the control acts on a neighborhood of the domain boundary.

- Note that this averaged observability result holds under, essentially, the condition that GCC is satisfied for the first equation. No assumption is done on the velocity of propagation of the second equation (other than being merely continuous and not coinciding with the first one in $\omega$ ).

- The assumption (2) requires the coefficients to be distinct everywhere in the observation subset. This assumption is not optimal since, for instance, the averaged observability inequality holds when $c_{1} \equiv c_{2}$ everywhere because, then, both solutions $u_{1}$ and $u_{2}$ coincide with its average. Obtaining sharp conditions on the coefficients for (3) to hold is an interesting open problem.

- The constant $C_{\theta}$ can be taken to be uniform for $\theta \geq \theta_{*}$ and $\theta_{*}>0$.

- The result can be easily generalized to a system with any finite number of modes, all starting from the same data. In that case the property (2) takes the form

$$
c_{1}(\mathbf{x})-c_{i}(\mathbf{x}) \neq 0, \quad \mathbf{x} \in \omega, i \neq 1,
$$

where $\omega$ satisfies the GCC in some time $T>0$ for the $i=1$. The coefficients are assumed to be continuous, except the leading one, $c_{1}$, which is assumed to be of class $\mathrm{C}^{1,1}$. Then for any averaging set of numbers $\theta_{i} \in[0,1], i=1, \ldots, N$ such that $\sum_{i} \theta_{i}=1$ and $\theta_{1}>0$ there exists a constant $C_{\theta}$ such that 


$$
E(0) \leq C_{\theta} \int_{0}^{T} \int_{\omega}\left|\sum_{i=1}^{\mathbf{N}} \theta_{i} \nabla u_{i}\right|^{2} d \mathbf{x} d t
$$

Proof: We now present the sketch of the proof that uses compactness-uniqueness arguments, similar as in $[3,6]$. We proceed in several steps.

Step 1. We first prove the following relaxed observability inequality with a compact reminder term:

Proposition 2.2 Under the assumptions of Theorem 2.1, for a suitable $C_{\theta}$, it holds:

$$
E(0) \leq C_{\theta}\left(\int_{0}^{T} \int_{\omega}\left|\theta u_{1}+(1-\theta) u_{2}\right|^{2} d \mathbf{x} d t+\left\|u^{0}\right\|_{\mathrm{H}^{-1}}^{2}+\left\|\tilde{u}^{0}\right\|_{\mathrm{H}^{-2}}^{2}\right) .
$$

Proof: We argue by contradiction. Assuming the contrary, there exist sequences of initial conditions $\left(u^{0 n}\right),\left(\tilde{u}^{0 n}\right)$, such that the corresponding solutions $u_{1}^{n}, u_{2}^{n}$ satisfy

$$
E^{n}(0)>n\left(\int_{0}^{T} \int_{\omega}\left|\theta u_{1}^{n}+(1-\theta) u_{2}^{n}\right|^{2} d \mathbf{x} d t+\left\|u^{0 n}\right\|_{\mathrm{H}^{-1}}^{2}+\left\|\tilde{u}^{0 n}\right\|_{\mathrm{H}^{-2}}^{2}\right) .
$$

Without loosing generality we can assume that $E^{n}(0)=1$.

Thus (5) implies that $\left\|u^{0 n}\right\|_{\mathrm{H}^{-1}}^{2}+\left\|\tilde{u}^{0 n}\right\|_{\mathrm{H}^{-2}}^{2} \rightarrow 0$, resulting in weak convergence of $\left(u^{0 n}, \tilde{u}^{0 n}\right) \longrightarrow(0,0)$ in $\mathrm{L}^{2}(\Omega) \times \mathrm{H}^{-1}(\Omega)$. Therefore the solutions $\left(u_{1}^{n}, u_{2}^{n}\right)$ converge weakly to zero in $\mathrm{L}^{2}(\langle 0, T\rangle \times \Omega)$ as well. In order to obtain a contradiction, we have to show that the last convergence is strong, at least for one component, $u_{1}^{n}$ or $u_{2}^{n}$, since this will imply the strong convergence to zero of the initial data, which is incompatible with the fact that $E^{n}(0)=1$.

From the contradictory assumption (5) we have that the H-measure $\nu$ associated to a subsequence of $\left(\theta u_{1}^{n}+(1-\theta) u_{2}^{n}\right)$ vanishes on $\langle 0, T\rangle \times \omega$. Furthermore, it is of the form

$$
\nu=\theta^{2} \mu_{1}+(1-\theta)^{2} \mu_{2}+\theta(1-\theta) 2 \Re \mu_{12}
$$

where, on the right hand side, the elements of the matrix measure associated to the vector subsequence of $\left(u_{1}^{n}, u_{2}^{n}\right)$ are listed, with $\mu_{12}$ denoting the off-diagonal element.

The continuity of coefficients enables an application of the localization property for H-measures (e.g. [9, Corollary 2.2]), which gives that each $\mu_{j}$ is supported within the corresponding characteristic set $\left\{\tau^{2}-c_{j}(\mathbf{x}) \boldsymbol{\xi}^{2}=0\right\}, j=1,2$. Due to the separation of velocities (2), it follows that their supports are disjoint on the observability region. On the other hand, because of the positive semi-definiteness of matrix $\mathrm{H}$-measures, off-diagonal entries are dominated by the corresponding diagonal elements. More precisely, it holds that $\operatorname{supp} \mu_{12} \subseteq \operatorname{supp} \mu_{1} \cap \operatorname{supp} \mu_{2}$, implying that $\mu_{12}=0$ on the observability region.

Thus we get that

$$
\nu=\theta^{2} \mu_{1}+(1-\theta)^{2} \mu_{2}=0 \quad \text { on }\langle 0, T\rangle \times \omega .
$$

As $\mu_{1}$ and $\mu_{2}$ are positive measures and $\theta>0$, it follows that $\mu_{1}$ vanishes on $\langle 0, T\rangle \times \omega$ as well. Using the regularity assumption on $c_{1}$ and taking into account that $\langle 0, T\rangle \times \omega$ satisfies the GCC for the problem (1) with $i=1$, the propagation property for H-measures (e.g. [11, Theorem 3.12]) gives that $\mu_{1}$ vanishes everywhere, implying strong convergence (to zero) of solutions $u_{1}^{n}$ in $\mathrm{L}^{2}((0, T) \times \Omega)$. The last convergence implies strong convergence to zero of initial data in $\mathrm{L}^{2}(\Omega) \times \mathrm{H}^{-1}(\Omega)$, and thus we get a contradiction to the assumption of the constant, non-zero initial energy.

Step 2. Here we follow a classical compactness-uniqueness procedure of reducing the observability for low frequencies to an elliptic unique continuation result [5].

Let $N(T)$ be a subspace of $\mathrm{L}^{2}(\Omega) \times \mathrm{H}^{-1}(\Omega)$, consisting of initial data for which the average of solutions to (1) vanishes on the observability region

$$
N(T):=\left\{\left(u^{0}, \tilde{u}^{0}\right) \in \mathrm{L}^{2}(\Omega) \times \mathrm{H}^{-1}(\Omega) \mid \theta u_{1}+(1-\theta) u_{2}=0, \text { on }\langle 0, T\rangle \times \omega\right\} .
$$


Based on the relaxed observability inequality (4) it follows that $N(T)$ is a finite dimensional space. Furthermore, the following characterization holds.

Lemma 2.3 $N(T)=\{0\}$.

Proof: Using that $N(T)$ is of finite dimension and the fact that the coefficients in the equations under consideration are time-independent, one gets that $N(T)$ contains a vector $\left(u^{0},-\lambda u^{0}\right)$, where $u^{0}$ is an eigenfunction of both Laplace operators $-\operatorname{div}\left(c_{i} \nabla\right), i=1,2$, under consideration, associated to a same eigenvalue $\lambda$.

By the definition of $N(T)$ it follows $\theta u_{1}(0)+(1-\theta) u_{2}(0)=u^{0}=0$ on $\omega$. Being an eigenfunction of the elliptic operator with $\mathrm{C}^{1,1}$ coefficient $c_{1}$, classical unique continuation results (e.g. [10, Theorem 3]) imply $u^{0}=0$ everywhere.

Step 3. Combining the two previous results, and using the contradiction argument in the same manner as in the proof of Proposition 2.2, one easily obtains the strong observability inequality (3).

The observability inequality (3) above is equivalent to a property of averaged control for a linear combination of solutions of the same wave system in the presence of a single control acting on all the components of the system simultaneously (see [12]):

$$
\begin{aligned}
\partial_{t t} v_{i}-\operatorname{div}\left(c_{i} \nabla v_{i}\right) & =\chi_{\langle 0, T\rangle \times \omega} f, \quad(t, \mathbf{x}) \in \mathbf{R}^{+} \times \Omega \\
v_{i}(0, \cdot) & =v_{i}^{0} \in \mathrm{H}^{1}(\Omega) \\
\partial_{t} v_{i}(0, \cdot) & =\tilde{v}_{i}^{0} \in \mathrm{L}^{2}(\Omega), \quad i=1,2,
\end{aligned}
$$

with $f \in \mathrm{L}^{2}\left(\mathbf{R}^{+} \times \Omega\right)$. More precisely, the following result holds.

Theorem 2.4 For any choice of initial data of the system (6), any positive constants $\alpha$ and $\beta$, and any final target $\left(v^{T}, \tilde{v}^{T}\right) \in \mathrm{H}^{1}(\Omega) \times \mathrm{L}^{2}(\Omega)$ there exists a control $f$ such that

$$
\left(\alpha v_{1}+\beta v_{2}\right)(T, \cdot)=v^{T}, \quad \partial_{t}\left(\alpha v_{1}+\beta v_{2}\right)(T, \cdot)=\tilde{v}^{T} .
$$

Note that unlike the system (1), the initial data for two components in (6) are not correlated. Remark 2

Proposition 2.2 also holds, under the same conditions of the coefficients $c_{1}$ and $c_{2}$ if, instead of the same initial data for the two equations entering in the system (1), one assumes that they are related by a bounded linear operator. However, in order to deduce the strong observability inequality (3), one has to adjust the proof of Lemma 2.3, which requires the unique continuation result to hold for an average of eigenfunctions associated to different elliptic operators. Such generalization can be obtained in two settings: either it is additionally assumed that coefficients $c_{i}$ are analytic and separated on the whole domain $\Omega$, or the linear operator linking the data of both solutions is such that whenever $\left(\left.\left(\theta u_{1}(0)+(1-\theta) u_{2}(0)\right)\right|_{\omega}=0\right)$ then $\left(\left.u_{1}(0)\right|_{\omega}=\left.u_{2}(0)\right|_{\mid \omega}=0\right)$. In addition, in the latter case, we have to require $\mathrm{C}^{1,1}$ regularity on all coefficients, to ensure the unique continuation for each elliptic equation.

By duality, this corresponds to controlling a suitable linear combination of both solutions.

\section{Simultaneous observability}

We reconsider the system (1) but with initial data depending on the parameter:

$$
\begin{aligned}
\partial_{t t} u_{i}-\operatorname{div}\left(c_{i}(\mathbf{x}) \nabla u_{i}\right) & =0, \quad(t, \mathbf{x}) \in \mathbf{R}^{+} \times \Omega \\
u_{i}(0, \cdot) & =u_{i}^{0} \in \mathrm{L}^{2}(\Omega) \\
\partial_{t} u_{i}(0, \cdot) & =\tilde{u}_{i}^{0} \in \mathrm{H}^{-1}(\Omega), \quad i=1,2 .
\end{aligned}
$$

Proceeding similarly as above, one obtains the following result. 
Theorem 3.1 Suppose the coefficients of (7) are analytic and satisfy $c_{1}(\mathbf{x})-c_{2}(\mathbf{x}) \neq 0, \mathbf{x} \in \Omega$. Let $\omega$ be an open subset of $\Omega$ that satisfies the GCC in some time $T>0$ for both problems in (1). Then, for any $\theta \in\langle 0,1\rangle$ there exists a constant $C_{\theta}$ such that the following estimate holds

$$
E_{1}(0)+E_{2}(0) \leq C_{\theta} \int_{0}^{T} \int_{\omega}\left|\theta u_{1}+(1-\theta) u_{2}\right|^{2} d \mathbf{x} d t
$$

As already stated in Remark 1, stronger assumptions on coefficients stated above are related to the unique continuation problem for an average $\theta u_{1}^{0}+(1-\theta) u_{2}^{0}$ of eigenfunctions associated to the elliptic operators considered in (7).

This result of simultaneous observability requires the GCC condition to be satisfied by both systems and not only by one of them as in the previous section. The corresponding dual problem consists of controlling each individual component of the system (6) by means of the same control. These results are closely related to those in $[1,6]$.

\section{The heat equation}

By using transmutation techniques as developed in [7] the results above can be employed to derive null controllability and observability properties for parameter-dependent parabolic equations as well:

$$
\begin{aligned}
\partial_{t} z_{i}-\operatorname{div}\left(c_{i}(\mathbf{x}) \nabla z_{i}\right) & =0, \quad(t, \mathbf{x}) \in \mathbf{R}^{+} \times \Omega \\
z_{i}(0, \cdot) & =z_{i}^{0} \in \mathrm{L}^{2}(\Omega), \quad i=1,2 .
\end{aligned}
$$

Using transmutations out of the solutions of the heat equations above we can define,

$$
u_{i}(s, \mathbf{x})=\int_{\mathbf{R}^{+}} k(t, s) z_{i}(t, \mathbf{x}) d t
$$

where the kernel $k$ is given by

$$
k(t, s)=\frac{1}{\sqrt{4 \pi t}} \sin \left(\frac{s S}{4 t}\right) e^{\frac{s^{2}-S^{2}}{4 t}},
$$

and $S>0$ is arbitrary, to get solutions $u_{i}$ of the wave equations with the same coefficients.

Applying the above results on simultaneous observation one gets the following:

Corollary 4.1 Assume the assumptions of Theorem 3.1 are satisfied. Then, for any $\tilde{T}>0$ and $\theta \in\langle 0,1\rangle$ there exists a constant $C_{\theta, \tilde{T}}$ such that the following estimate holds

$$
\sum_{i} \int_{0}^{\infty} e^{-\frac{S^{2}}{2 t}}\left\|z_{i}(t, \cdot)\right\|_{\mathrm{L}^{2}}^{2} d t \leq C_{\theta, \tilde{T}} \int_{0}^{\tilde{T}} \int_{\omega}\left|\left(\theta z_{1}+(1-\theta) z_{2}\right)(t, \mathbf{x})\right|^{2} d \mathbf{x} d t
$$

This corollary enables one to estimate the energy of the system (8) at an arbitrary strictly positive time, namely, $\left\|z_{1}(\tilde{T}, \cdot)\right\|_{\mathrm{L}^{2}}+\left\|z_{2}(\tilde{T}, \cdot)\right\|_{\mathrm{L}^{2}}$. However, its initial energy at time $t=0$ can not be recovered from (9) because of the time-irreversibility of the heat equations under consideration.

The assumption on the set $\omega$ to satisfy the GCC could seem to be unnatural, since for scalar heat equations no geometric conditions are needed for observability/controllability to hold. But this is the first result in this direction and it does not fit in the existing literature of simultaneous control of heat like equations where, most often, the principal part of the differential equations entering in the heat equations of the system are assumed to be the same (see [2]). 


\section{Conclusion}

The results presented above can also be generalized to the case where the system under consideration involves an infinite number of equations depending on a parameter, either discrete, or even continuous. Such generalizations are not straightforward and one has to pay special attention to the arguments based on the localization property for $\mathrm{H}$-measures. In particular, when averaging an infinite number of sequences, the measure associated to the average does not have necessarily to be supported within the set containing the supports of all H-measures associated to each particular sequence, which disables the arguments used in the proof of Proposition 2.2. Similarly, the condition on the separation of velocities requires more detailed analysis. This issue will be analyzed in a forthcoming work.

The results we have presented on parabolic equations are the first ones of that type. It would be interesting to see if Carleman inequalities, which is the tool that is most efficiently used to analyze observability problems for parabolic equations (see [8]), can be directly applied to address these issues.

\section{Acknowledgements}

This paper has mainly been developed while the first author was a Postdoctoral Fellow on the Basque Center for Applied Mathematics (Bilbao, Spain) within the NUMERIWAVES FP7-246776 project. Also supported in part by the Ministry of Science, Education and Sports of the Republic of Croatia (project 037-0372787-2795). This work was finished while the second author was visiting the Laboratoire Jacques Louis Lions with the support of the Paris City Hall "Research in Paris" program. E. Zuazua was also partially supported by Grants MTM2008-03541 and MTM2011-29306 of MICINN Spain, Project PI201004 of the Basque Government, ERC Advanced Grant FP7-246775 NUMERIWAVES and ESF Research Networking Programme OPTPDE.

The authors acknowledge Belhassen Dehman for a fruitful discussion, and the referee for the remarks that have improved the final version of the paper.

\section{References}

[1] F. Alabau-Boussouira, M. LÉautaud, Indirect controllability of locally coupled wave-type systems and applications, J. Math. Pures Appl. 99(5) (2013) 544-576.

[2] F. Ammar-Khodja, A. Benabdallah, M. GonzÁlez-Burgos, L. De Teresa, Recent results on the controllability of linear coupled parabolic problems: a survey, Math. Control Relat. Fields 1(3) (2011) 267-306.

[3] N. Burq, Contrôle de l'équation des ondes dans des ouverts peu réguliers, Asymptot. Anal. 14(2) (1997) $157-191$.

[4] N. Burq And P. GÉrard, Condition nécessaire et suffisante pour la contrôlabilité exacte des ondes, C. R. Acad. Sci. Paris Sér. I Math. 325(7) (1997) 749-752.

[5] C. Bardos, G. Lebeau, J. Rauch, Sharp sufficient conditions for the observation, control, and stabilization of waves from the boundary, SIAM J. Control Optim. 30(5) (1992) 1024-1065.

[6] B. Dehman, M. Léautaud, J. Le Rousseau, Controllability of two coupled wave equations on a compact manifold, Arch. Rational Mech. Anal. 211(1) (2014) 113-187.

[7] S. Ervedoza, E. ZuazuA, Sharp observability estimates for heat equations, Arch. Rational Mech. Anal. 202(3) (2011) 975-1017.

[8] E. Fernández-Cara, E. Zuazua, The cost of approximate controllability for heat equations: The linear case, Adv. Differential Equations 5(4-6) (2000) 465-514.

[9] P. GÉrard, Microlocal Defect Measures, Comm. Partial Differential Equations 16(11) (1991) $1761-1794$. 
[10] L. Hörmander, On the uniqueness of the Cauchy problem. II., Math. Scand. 7 (1959) 177-190.

[11] L. TARTAR, H-measures, a new approach for studying homogenisation, oscillation and concentration effects in PDEs, Proc. Roy. Soc. Edinburgh. Sect. A 115(3-4) (1990) 193-230.

[12] E. ZuazuA, Averaged control, preprint, 2013. 